\title{
TUBERCULOSIS IN NSW: STATUS AND PRIORITIES
}

Timothy Heath $h^{1,3}$, Meg Winks', Christine Roberts ${ }^{3}$, Anthony Capon ${ }^{1}$

1. Western Sector Public Health Unit

2. Formerly AIDS/Infectious Diseases Branch, NSW Health Department

3. National Centre for Epidemiology and Population Health, Australian National University, ACT

$\mathrm{T}$

his article provides a profile of tuberculosis occurrence in

NSW, based on an analysis of surveillance data for 1995 .

TB has re-emerged globally as a major threat to human health. It now causes more deaths worldwide than any other infectious disease, and is responsible for a quarter of preventable adult deaths worldwide ${ }^{1,2}$. Abetted by HIV/AIDS, the brunt of TB disease is borne by the developing world ${ }^{3}$. However, the USA, the UK and several European countries have also encountered increasing rates of active TB ${ }^{4}$. Outbreaks of multi-drug-resistant TB in urban and hospital settings in the USA have posed the additional threat of untreatable disease ${ }^{5}$. While TB rates in NSW (as in most other Australian States) have remained among the world's lowest, there is no room for complacency.

\section{METHODS}

NSW TB notification data for 1995 were obtained from the NSW Health Department's Infectious Diseases Surveillance System (IDSS) database for notifiable infectious diseases. Australian Bureau of Statistics (ABS) 1991 census data were used to calculate disease rates by country of birth and Aboriginality. All other rates were calculated using ABS estimated mid-year populations.

\section{Definitions}

In NSW, the surveillance definition of active TB (for notification purposes) is:

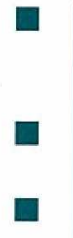

signs and symptoms compatible with pulmonary $\mathrm{TB}$, and an abnormal, unstable chest x-ray, (i.e. one which suggests disease progression); $O R$ signs and symptoms compatible with extrapulmonary TB; OR evidence of disease where treatment with two or more anti-TB drugs have been prescribed; OR
TABLE 1

TUBERCULOSIS NOTIFICATIONS BY SITE OF DISEASE, NSW, 1995

\begin{tabular}{|llrr|}
\hline Site of disease & & $\begin{array}{r}\text { No of } \\
\text { cases }\end{array}$ & $\%$ \\
\hline Pulmonary & Respiratory & 240 & $(52)$ \\
& Primary & 20 & $(4)$ \\
\hline Extrapulmonary & Genito-urinary & 22 & $(5)$ \\
& Bone/joint & 20 & $(4)$ \\
& Gastrointestinal & 9 & $(2)$ \\
& CNS/meningitis & 4 & $(1)$ \\
& Miliary & 4 & $(1)$ \\
& Other (incl. lymphatic) & 119 & $(26)$ \\
\hline Not specified & & 24 & $(5)$ \\
\hline Total & & 462 & $(100)$ \\
\hline
\end{tabular}

isolation of Mycobacterium tuberculosis complex organisms from a clinical specimen; OR demonstration of acid fast bacilli (AFB) in a clinical specimen from a person with signs and symptoms compatible with pulmonary TB.

A new case of active TB is an individual who has not previously received anti-TB chemotherapy for more than one month. A reactivated case is one where active TB has recurred more than one year after completion of therapy for proven disease.

Treatment default is defined as interruption of treatment for more than two months, after completing the first month of chemotherapy.

\section{RESULTS}

\section{Disease classification and site}

By August 1996, 462 cases of active TB had been reported in NSW with onset in 1995 ( $7.5 / 100,000$ population). Of these, 334 notifications ( 72 per cent) were new cases, 34 ( 7 per cent) were reactivated cases, and for 95 ( 21 per cent) a case classification was not specified. The principal anatomical site of disease was extrapulmonary for 178 notifications (38 per cent) (Table 1 ).

Continued on page $136 \mathrm{D}$
(CHATA) has supported TB-related research in Australia (through the Harry Windsor Scholarship scheme) and TB control activities in Asia - particularly Vietnam. The Public Health Association of Australia convened the National Tuberculosis Conference in November 1994 and is planning a second meeting during 1997.

CHATA's support of TB control activities in Asia is part of Australia's increasing recognition of its regional responsibilities in TB control. Since 1995, the Australian Government, through the Australian Agency for International Development, has supported TB control efforts in Indonesia and throughout the Pacific region under the World Health Organization's Global Tuberculosis Program. Australian-trained public health professionals are guiding TB control activities in Indonesia, China and
Mongolia. Australians have been prominent in working with refugees in Hong Kong and Vietnam; guidelines for control of TB among refugees and displaced persons are being devised through the Macfarlane Burnet Centre for International Health; and economic analyses of TB control activities in China are being undertaken through the National Centre for Epidemiology and Population Health at the Australian National University in Canberra. Australian laboratories are participating in a global network for the monitoring of Mycobacterium tuberculosis drug resistance.

1. Dwyer B. What we should be doing about tuberculosis in Australia. Med J Aust 1996; 164:62-63.

2. Gilbert GL. Multi-drug-resistant tuberculosis: prevention is better than cure. Med J Aust 1996; 164:121-124.

3. Hargraves J. Tuberculosis notifications in Australia, 1993. Commun Dis Intell 1995; 19:334-343. 


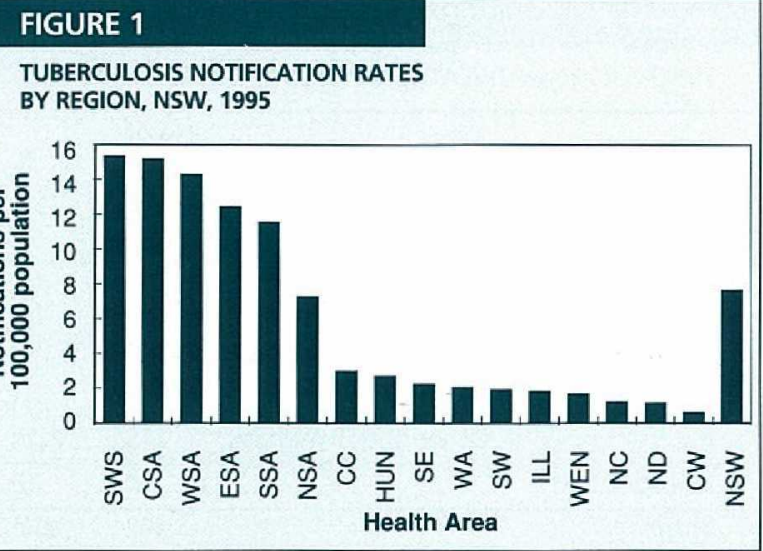

CSA = Central Sydney Area; ESA = Eastern Sydney Area; SWS = South Western Sydney; SSA = Southern Sydney; WSA = Western Sydney Area; NSA = Northern Sydney Area; ILL = Illawarra; ND = Northern Districts; HUN = Hunter; SW = South Western; North Coast; WEN = Wentworth; $\mathrm{CC}=$ Central Coast; WA = Western Area; $\mathrm{SE}=$ South Eastern; $\mathrm{CW}=$ Central West; NSW $=$ NeW South Wales.

\section{FIGURE 2}

TUBERCULOSIS NOTIFICATION RATES

BY AGE AND SEX, NSW, 1995

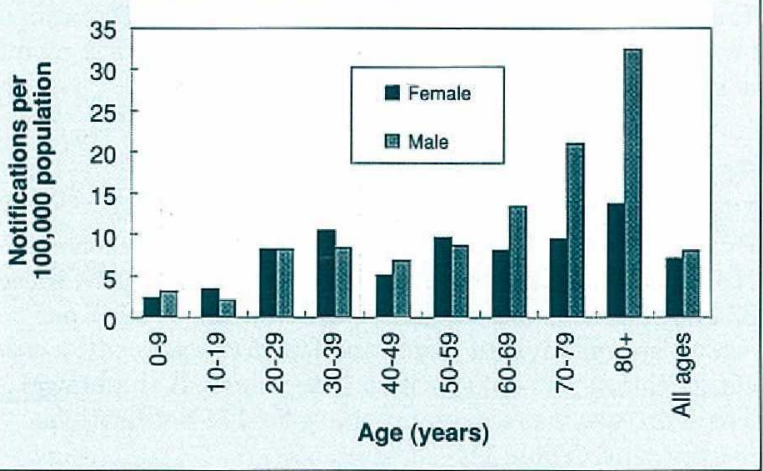

\section{FIGURE 3}

TUBERCULOSIS NOTIFICATIONS BY AGE

AND PLACE OF BIRTH, NSW, 1995

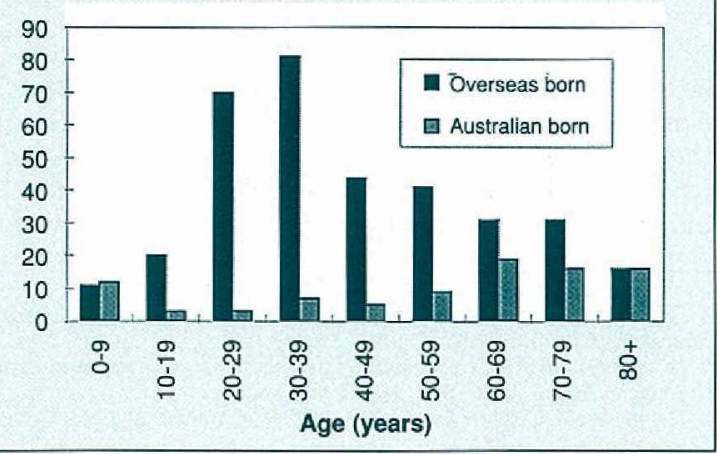

Tuberculosis in NSW

Continued from page 135

Infectivity and drug resistance

A sputum or bronchoscopic microscopy result was reported for 178 of the 260 pulmonary cases ( 68 per cent), and of these 97 (54 per cent) were positive for AFB on direct smear. Sputum from 85 pulmonary cases was direct smear positive on at least one occasion. A culture result was reported for 324 of all TB notifications ( 70 per cent), and 243 of these ( 75 per cent) were culture positive -241 for $M$. tuberculosis, and two for $M$. bovis.

Fourteen isolates ( 6 per cent of culture positive notifications) were reported as isoniazid-resistant. Three isolates were resistant to pyrazinamide, including two $M$. bovis isolates which are intrinsically resistant to pyrazinamide. Only one isolate was resistant to both isoniazid and rifampicin.

Demographic characteristics of people notified with TB TB notification rates were much higher in the Sydney metropolitan area than in rural NSW (Figure 1).

Notification rates were highest among the elderly, but a smaller peak occurred in the 20-40 year age range (Figure 2). The peak in young adults largely represented overseasborn cases (median age 38 years), while most Australianborn cases notified were in older age groups (median 65 years) (Figure 3). The sex distribution for TB notifications was approximately equal (male:female $=1.1: 1.0$ ).

Country of birth was reported for 435 TB notifications in NSW (94 per cent), and of these 345 ( 79 per cent) were of people born overseas. The notification rate for the overseasborn population in NSW was thus $26.3 / 100,000$, compared to 2.1 notifications per 100,000 Australian-born people, and 4.3/100,000 Aboriginal and Torres Strait Island people. Language spoken at home was recorded for 420 people and 53 per cent of these were from a non-English speaking background. Time since arrival in Australia was reported for 285 overseas-born cases ( 83 per cent), and of these more than 80 per cent were notified more than $2 \frac{1}{2}$ years after immigration (median six years; range one month to 46 years).

\section{HIV-TB}

HIV status was reported for 25 TB notifications (5 per cent of the total). Six were HIV seropositive, and two of these were Australian-born. All the $M$. tuberculosis isolates cultured from people with HIV-TB co-infection were fully drug-susceptible.

\section{DISCUSSION}

During the past 10 years, the annual number of TB notifications in NSW has increased steadily by more than half to 462 cases, from 290 cases in $1986^{6}$. The factors underlying this increase are complex. They reflect changes in population structure, including age structure, and infection acquired in high-prevalence countries. This has resulted in increased demands on available TB services, particularly in metropolitan Sydney where most TB notifications occur.

In NSW, TB is predominantly an imported disease, and consequently TB services must serve a wide spectrum of non-English speaking backgrounds and cultural beliefs. This places demands on available services, because 
culturally appropriate explanation and language services are essential if optimal compliance with TB treatment and screening is to be achieved.

So far, TB notification rates for Australian-born residents in NSW have remained very low, suggesting either that transmission is not occurring between immigrant and Australian-born populations, or that transmission has not yet expressed itself as active disease. A recent crosssectional Mantoux survey of children at school entry in Central, Southern and South Western Sydney found that 2.8 per cent of Australian-born children were Mantoux positive (diameter of induration $\geq 10 \mathrm{~mm}$ ) compared with 17.8 per cent of overseas born children. Mantoux positivity was much lower among Australian-born children whose parents were born overseas ( 3.0 per cent) than for overseasborn children ${ }^{7}$. Of Australian-born children whose parents were also born in Australia, 2.1 per cent were Mantoux positive.

A previous survey in the same region found that 2 per cent of year 8 Australian-born school children were Mantoux positive, similar to that found in the school entry study ${ }^{8}$. Thus children are at greater risk of TB infection if they were born overseas than if they were born to immigrant families in Australia. It also appears that relatively little TB transmission is occurring between overseas-born and Australian-born school children, perhaps because children with active TB are not usually contagious. Less is known about the extent of TB transmission between immigrant and Australian-born adult populations in NSW.

Drug resistance has not yet emerged as a major problem in NSW. One of the important measures designed to prevent drug resistance is directly observed therapy (DOT). To maintain drug susceptibility, resources must be made available for DOT, and DOT programs must be implemented effectively.

Although case finding and DOT are the first priorities for TB control, screening high-risk groups is also important in populations with low TB endemicity such as the NSW population". The most important screening programs are contact tracing and immigrant screening, but enhanced surveillance is needed for other groups at high risk for TB. Information on these groups is sub-optimal. They include people living with HIV-AIDS, injecting drug users, the homeless, prison inmates, others living in institutions on a long-term basis, hospitalised patients and health care workers. Prompt notification and contact tracing in these settings is critical, because these groups are particularly prone to clusters of TB disease.
Preventive therapy (chemoprophylaxis) can greatly reduce the incidence of active disease in TB-infected people, and consequently it is an important component of TB screening ${ }^{10}$. With immigrant screening, a minority of those screened develop active disease during the $2 \frac{1}{2}$-year observation period following arrival in Australia. Therefore this observation period is unlikely to contribute greatly to TB case finding and prevention, unless preventive therapy is also promoted as an integral part of immigrant screening.

Further analysis of surveillance data is required to determine the relative contributions of the factors underlying the observed increase in TB notifications over the past decade. There are more cases of TB in NSW than in any other Australian State, so our approach to TB surveillance and control will be pivotal to the Australian response $^{11}$.

\section{ACKNOWLEDGMENTS}

We thank Gary Burns (Occupational Health and Safety Unit, Western Sydney Area Health Service) for assisting with the preparation of this report. Tim Heath's Master of Applied Epidemiology position is jointly funded by the Western Sector Public Health Unit and the AIDS/Infectious Diseases Branch of the Commonwealth Department of Health and Family Services.

1. Murray CJL, Styblo K, Rouillon A. Tuberculosis in developing countries: burden, intervention and cost. Bull Int Union Tuberc Lung Dis 1990; 65:5-24.

2. Godlee F. Tuberculosis - a global emergency. Br Med J 1993; 306:1147.

306:1147. Krause VL, Britton WJ. Tuberculosis in the tropics. Med J Aust 1993; 159:412-415.

4. Murray JF. The International Union Against Tuberculosis and Lung Disease: its contribution to world lung health. Am J Respir Crit Care Med 1995; 151:1697-1699.

5. Iseman MD. Treatment of multi-drug-resistant tuberculosis. New Engl J Med 1993; 329:784-791.

6. Levy M. Annual tuberculosis report. NSW Health 1986.

7. Alperstein G, Morgan K, Fett MJ, Thomas M, Nossar V, Stewart G. Prevalence of tuberculosis infection among primary school entry children in Sydney. Aust eJ Public Health 1996; 20:123-128.

8. Alperstein G, Fett MJ, Reznik R, Thomas M, Senthil M. Prevalence of tuberculosis infection among year 8 school children in inner Sydney in 1992. Med J Aust 1994; 160:197-201.

9. Centers for Disease Control. Essential components of a tuberculosis prevention and control program; and screening for tuberculosis and tuberculosis infection in high-risk populations: recommendations of the Advisory Council for the Elimination of Tuberculosis. MMWR Morb Mortal Whly Rep 1995; 44:1-34.

10. Centers for Disease Control. Screening for tuberculosis and tuberculosis infection in high-risk populations; the use of preventive therapy for tuberculosis infection in the United States. MMWR Morb Mortal Whly Rep 1990; 39:RR-8.

11. Oliver G. Tuberculosis notifications in Australia, 1994. Comm Dis Intell 1996; 20:108-115. 